УДК 517.55, 512.628.2, 517.589

\title{
On Analytical Complexity of Antiderivatives
}

\author{
Maria A. Stepanova* \\ Steklov Mathematical Institute RAS \\ Gubkina, 8, Moscow, 119991
}

Russia

Received 29.07.2019, received in revised form 04.09.2019, accepted 20.10.2019

It is shown that the class of all functions of two variables of finite analytical complexity is not closed under integration. It also follows that the class of all functions of finite analytical complexity in the case of three or more variables is not closed under integration. For the case of three or more variables explicit examples of finite complexity functions with infinite complexity antiderivatives are constructed.

Keywords: analytical complexity, integration, finite complexity functions. DOI: 10.17516/1997-1397-2019-12-6-694-698.

Let $f(x, y)$ be a holomorphic function in a polydisc $G$. Denote by $\triangle$ the mapping of $f$ to its partial derivative with respect to $x$. Denote by $I$ the mapping of $f$ to one arbitrarily chosen from the class of all antiderivatives of the function $f$ antiderivative with respect to $x$.

To choose an antiderivative we can, for instance, integrate the function $f$ with respect to $x$ along a path with the beginning in the center of the polydisc $G$.

Let us extend the mappings $\triangle$ and $I$ to analytical functions. To define the mapping $I$ we pick a germ of an analytical function $F(x, y)$ at some point and consider its representative in the polydisc. For the representative we construct an antiderivative with the help of the mapping $I$ and then continue the obtained antiderivative along all the paths, along which the continuation of the initial function is possible.

Remark 1. Note that all analytic antiderivatives of an analytic function $F$ differ by an analytic function depending on the variable $y$ (because an antiderivative in the polydisc in which the function is represented by its Taylor series is defined up to a function of the variable $y$ which depend on the initial point of the path of integration). It follows that all analytic antiderivatives of $F$ have simultaneously either finite analytical complexity (which differs not more than by one for any two antiderivatives of $F$ ) or infinite analytical complexity.

Remark 2. We can also define mappings similar to $\triangle$ and $I$ for the variable $y$, and further without loss of generality we consider only the case of the variable $x$.

Let $\mathbf{C l}$ be the class of all functions of finite analytical complexity and let $C l_{\infty}$ be the class of all functions of infinite analytical complexity. From the chain rule it follows that $\triangle\left(C l_{n}\right) \subset C l_{2 n}$ ( [1]) from which we obtain that $\triangle(\mathbf{C l}) \subset \mathbf{C l}$, i.e. the class of all functions of finite complexity is closed under taking derivatives.

In [2] the question about the change of complexity of functions after integration was posed. Similar questions about elementary functions (i.e. is the antiderivative of an elementary function also elementary) were considered in $[3,4]$.

The aim of this paper is to show that the class $\mathbf{C l}$ is not closed under integration:

*step_masha@mail.ru

(c) Siberian Federal University. All rights reserved 


\section{Theorem. $I(\mathbf{C l}) \not \subset \mathbf{C l}$.}

Remark 3. Arguing by contradiction, from the inclusion $\triangle(\mathbf{C l}) \subset \mathbf{C l}$ we deduce that $I\left(\mathrm{Cl}_{\infty}\right) \subset$ $I\left(C l_{\infty}\right)$ and from the statement of the theorem we deduce that $\triangle\left(C l_{\infty}\right) \not \subset C l_{\infty}$.

For the proof of the theorem we need some auxiliary considerations.

Let $D_{n}(f)=0$ be the differential criterion of belonging to the class $C l_{n}$ for the function $f$. The existence of this criterion is prooved in [5] (see also [6]).

Let us formulate properties of the system $D_{n}(f)=0$ necessary for later use:

1) $D_{n}(f)=0$ is a system of polynomial equations with integer coefficients for derivatives $\frac{\partial^{n+m} f}{\partial x^{n} \partial y^{m}}$ of $f$

2) the equations of the system $D_{n}(f)=0$ do not depend on $f$ as a variable.

Lemma 1. For any integer nonnegative number $M$ there exists a polynomial of complexity $M$.

Proof. First, observe that there exist polynomials of arbitrarily high complexity. Indeed, all equations of the system $D_{n}(f)=0$ have finite order, and therefore a generic polynomial of sufficiently high degree does not satisfy the equations of this system.

Pick a polynomial $P(x, y)$ of complexity $\tilde{M}, \tilde{M} \geqslant M$. In what follows, we assume that $M \geqslant 1$, because in the case $M=0$ the desired polynomial is a polynomial of one variable. Let us number all monomials of the polynomial $P$ in an arbitrary manner. Since after subtracting a monomial the complexity of a function cannot change by more than one, subtracting successively all monomials from $P$ in accordance with the chosen numeration, we obtain the sequence of polynomials of non-increasing complexity, in which for any $j, 1 \leqslant j \leqslant \tilde{M}$, there exists a polynomial of complexity $j$. By the inequality $1 \leqslant M \leqslant \tilde{M}$ we obtain the assertion of Lemma 1 .

Proposition. For all $n$ there exist a function $g(x, y)$ of complexity $n$ and analytical functions of one variable $a(x), b(y)$, such that the function $\tilde{g}(x, y)=g(x, y)+a(x)+b(y)$ has complexity $n+1$.

Proof. Assume the contrary: suppose that for all functions $a(x), b(y)$ and any function $g(x, y)$ of complexity $n$ the function $\tilde{g}(x, y)$ also has complexity $n$. Then since for any nonconstant function $c(z)$ functions $\tilde{g}, c \circ g$ and $c \circ \tilde{g}$ have the same complexity, choosing as $a, b, c$ the functions $\ln \left(x^{\alpha}\right), \ln \left(y^{\beta}\right), \exp (z)$, respectively, we obtain that the multiplication of the function $g$ by the monomial $x^{\alpha} y^{\beta}$ also does not change the complexity. But in this case it is possible to do one after another $K$ times the following two operations for the function $g \cdot y^{-K}$ without changing the complexity:

1) adding of an arbitrary polynomial of $x$ of degree $K$,

2) multiplying by $y$,

and then add an arbitrary polynomial of the variable $x$ and an arbitrary polynomial of the variable $y$. Consequently, the complexity does not change after adding an arbitrary polynomial of two variables to the function $g$. But by Lemma 1 there exist polynomials of arbitrarily high complexity. It follows that described operations result in the growth of the complexity. Since by Lemma 1 the class of all functions of complexity $n$ is nonempty, we arrive at a contradiction, which completes the proof.

Let $\left\{e q_{1}=0, \ldots, e q_{k(n)}=0\right\}$ be the equations of the system $D_{n}(f)=0$.

Lemma 2. The system $D_{n}(f)=0$ essentially depends on $f_{x}:=\frac{\partial f}{\partial x}$, i.e. there exists $j$, $1 \leqslant j \leqslant k(n)$, such that $\frac{\partial\left(e q_{j}\right)}{\partial\left(f_{x}\right)} \not \equiv 0$. 
Proof. Assume the contrary. Since the equations of the system $D_{n}(f)=0$ do not depend on $f$ as a variable (property 2) and do not depend on $f_{x}$ by our assumption (note that due to the symmetry of the equations $D_{n}(f)=0$ in relation to $x$ and $y$ these equations do not depend also on $f_{y}$ ), for all functions $g(x, y)$ functions $g$ and $\tilde{g}=g+x+y$ have the same complexity. But it is impossible in view of the Statement above, because the transformation $(x, y) \longrightarrow(a(x), b(y))$ does not change the complexity.

The proof of Lemma 2 is complete.

Consider the set $C l_{n}(G)$ of functions from the class $C l_{n}$, such that they have a holomorphic representative (i.e. an element) in a common for all functions polydisc $G . C l_{n}(G)$ is a metric space as a subset of the Fréchet space $\mathcal{O}(G)$ of all functions holomorphic in the polydisc $G$.

Lemma 3. For any $n$ the space $C l_{n}(G)$ is complete in the topology of uniform convergence on compact subsets of $G$.

Proof. Let us show that the subset $C l_{n}(G)$ of the space $\mathcal{O}(G)$ is closed, from which in view of the completeness of the space $\mathcal{O}(G)$ the completeness of the space $C l_{n}(G)$ follows. If $f_{k} \longrightarrow f$, where $f_{k} \in C l_{n}(G)$, then by the Weierstrass theorem all derivatives of the functions $f_{k}$ also converge to the derivatives of $f$, and therefore from the identities $D_{n}\left(f_{k}\right)=0$ the identity $D_{n}(f)=0$ follows.

The proof of Lemma 3 is complete.

Proof of the Theorem. It suffices to show that there exists a holomorphic in the polydisc $G$ function of finite complexity, such that its antiderivative with respect to $x$ has infinite complexity. Assume the contrary: let all antiderivatives of functions of finite complexity also be of finite complexity. Suppose that the mapping $I$ sends the function $h_{x}$ into $h$ and suppose that $h_{x} \in$ $C l_{n}(G)$. The criterion of belonging to the class $C l_{n}(G)$ is $D_{n}\left(h_{x}\right)=0$. By our assumption, for any function $h_{x} \in C l_{n}(G)$ there exists the number $m=m\left(h_{x}\right)$, such that $h \in C l_{m}(G)$, or, equivalently, $D_{m}(h)=0$. Hence we obtain that

$$
C l_{n}(G)=\bigcup_{m} \bigcup_{h \in C l_{m}(G), m=m\left(h_{x}\right)} h_{x} .
$$

Note that the system $D_{n}\left(h_{x}\right)=0$ does not depend on $h_{x}$ as a variable (property 2), while by Lemma 2 the system $D_{m}(h)=0$ depends on $h_{x}$ as a variable. Consequently, equations of the system $D_{m}(h)=0$ determine a nowhere dense subset in the set $H(G)$ of all holomorphic in $G$ solutions $h_{x}$ of the system $D_{n}\left(h_{x}\right)=0$ in the topology induced from the topology of uniform convergence, since for any open neighbourhood it is possible to vary the value of the derivative $h_{x}$ at any fixed point, values of the other derivatives being fixed. But by Lemma 3 the space $C l_{n}(G)$ is complete, consequently, it is impossible to represent it as a countable union of nowhere dense sets, which contradicts our assumption. Therefore there exist solutions $h_{x}$, such that the function $h$ has infinite complexity.

The proof of the Theorem is complete.

For analytical functions $f\left(x_{1}, \ldots, x_{N}\right)$ of larger number of variables it is possible to construct a similar system of classes $C l_{n}(N)([7])$. Let $\mathbf{C l}(N)$ be the class of all functions in $N$ variables of finite complexity. From the Theorem we obtain

\section{Corollary 1. $I(\mathbf{C l}(N)) \not \subset \mathbf{C l}(N)$.}

Proof. It suffices to consider a function $f\left(x_{1}, x_{2}, \ldots, x_{N}\right)$ as a function of two variables $x_{1}, x_{2}$ with parameters $x_{3}, \ldots, x_{N}$. 
The proof of the Corollary is complete.

For the case $N>2$ it is possible to give concrete examples of functions of finite complexity, such that after integration the complexity becomes infinite.

Example 1. Case $N=3$.

Consider the solution of the heat equation $u_{y}=u_{x x}$ of infinite complexity:

$$
u(x, y)=\sum_{r=1}^{\infty} e^{-\sqrt{p_{r}}} e^{r x+r^{2} y}
$$

where $p_{r}$ is $r$-th prime number.

We write $u(x, y)$ in the form of the Poisson integral:

$$
u(x, y)=\frac{1}{2 \sqrt{\pi y}} \int_{-\infty}^{\infty} \exp \left(-\frac{(x-\zeta)^{2}}{4 y}\right) \varphi(\zeta) d \zeta,
$$

where $\varphi(\zeta)$ is the initial function.

Observe that the complexity of the integrand is finite and is equal to two. Indeed, firstly, the complexity of this function is greater than one, since its complexity as the function of two variables $(y, \zeta)$ equals two, the value of $x$ being fixed (this can be verified by substituting the function under consideration in the differential criterion of belonging to the first complexity class), and, secondly, the complexity of this function is not greater than two.

Consider the functions $U_{-}(x, y, z):=\frac{1}{2 \sqrt{\pi y}} \int_{-\infty}^{z} \exp \left(-\frac{(x-\zeta)^{2}}{4 y}\right) \varphi(\zeta) d \zeta$ and $U_{+}(x, y, z):=$ $\frac{1}{2 \sqrt{\pi y}} \int_{z}^{\infty} \exp \left(-\frac{(x-\zeta)^{2}}{4 y}\right) \varphi(\zeta) d \zeta$. Since $U_{-}+U_{+}=u$ holds, at least one (an hence both due to the symmetry) of the functions $U_{-}, U_{+}$has infinite complexity.

Thus, any of the two functions $U_{-}, U_{+}$gives the desired example.

Example 2. Case $N>3$.

Let us add to the functions $U_{-}, U_{+}$from Example 1 above $N-3$ parameters (multiplying, for instance, by $N-3$ variables $): V_{ \pm}\left(x_{1}, x_{2}, x_{3}, x_{4}, \ldots, x_{N}\right):=U_{ \pm}\left(x_{1}, x_{2}, x_{3}\right) \cdot \prod_{j=4}^{N} x_{j}$.

The author is grateful to V.K. Beloshapka for fruitful discussions.

This work is supported by the Russian Science Foundation under grant 19-11-00316.

\section{References}

[1] V.K.Beloshapka, Analytical complexity: Development of the topic, Russian Journal of Mathematical Physics, 19(2012), no. 4, 428-439.

[2] V.K. Beloshapka, On Integration of Functions of Complexity One, Journal of Siberian Federal University. Mathematics \& Physics, 12(2019), no. 4, 1-7.

[3] J.Liouville, Sur la détermination des intégrales dont la valeur est algébrique, Journal de l'ecole polytechnique, XIV(1833), Sec. 23.

[4] J.F.Ritt, Integration in finite term, Liouville's theory of elementary methods, NY, Colombia University Press, 1948.

[5] V.K.Beloshapka, Decomposition of functions of finite analytical complexity, Journal of Siberian Federal University. Mathematics \& Physics, 11(2018), no. 6, 680-685. 
[6] V.K. Beloshapka, Analytic complexity of functions of two variables, Russian Journal of Mathematical Physics, 14(2007), no. 3, 243-249.

[7] V.K. Beloshapka, Analytic Complexity of Functions of Several Variables, Mat. Zametki, $\mathbf{1 0 0}(2016)$, no. 6, 781-789.

\title{
Об аналитической сложности первообразных
}

\author{
Мария А. Степанова \\ Математический институт им. В. А. Стеклова РАН \\ Губкина, 8, Москва, 119991 \\ Россия
}

Показано, что класс всех функиий двух переменнъх конечной аналитической сложности не замкнут относительно операции интегрирования. Отсюда следует также и незамкнутость относительно операчии интегрирования класса всех функиий конечной аналитической сложности для трех и большего числа переменных. Для случая трех и большего числа переменных приведены конкретные примеры функиий конечной сложсности, первообразные которых имеют бесконечную сложность.

Ключевые слова: аналитическая сложность, интегрирование, функиии конечной сложности 